\title{
Soft Gluon Resummation and a Novel Asymptotic Formula for Double-Spin Asymmetries in Dilepton Production at Small Transverse Momentum
}

\author{
Hiroyuki Kawamura $^{1}$, Jiro Kodaira ${ }^{2 *}$ and Kazuhiro Tanaka ${ }^{3}$ \\ 1- Radiation Laboratory, RIKEN, Wako 351-0198, Japan \\ 2- Theory Devision, KEK, Tsukuba 305-0801, Japan \\ 3- Department of Physics, Juntendo University, Inba, Chiba 270-1695, Japan
}

\begin{abstract}
We discuss the double-spin asymmetries $\mathcal{A}_{T T}\left(Q_{T}\right)$ in transversely polarized Drell-Yan process at small transverse momentum $Q_{T}$ of the produced dilepton. Soft gluon radiations relevant for small $Q_{T}$ are resummed to all orders in $\alpha_{s}$, up to the next-to-leading logarithmic accuracy. We show that the soft gluon contributions to polarized and unpolarized cross sections mostly cancel in the asymmetries, but significant corrections still remain. We propose a novel asymptotic formula for $\mathcal{A}_{T T}\left(Q_{T}\right)$ at small $Q_{T}$, which provides a new approach to extract the transversity $\delta q(x)$ from the experimental data.
\end{abstract}

Transversly polarized Drell-Yan (tDY) process, $p^{\uparrow} p^{\uparrow} \rightarrow l^{+} l^{-} X$, is one of the processes where we can measure the chiral-odd transversity distributions, $\delta q(x)$. The NLO cross sections of tDY, with the transverse momentum $Q_{T}$ of the final dilepton unobserved (integrated), has been studied at RHIC kinematics in [2], and it turned out that the corresponding double transverse-spin asymmetries $A_{T T}$ are small because, at RHIC, the sea-quark distributions are probed at small partonic momentum fraction $x$. Here, we consider the double-spin asymmetries $\mathcal{A}_{T T}\left(Q_{T}\right)$ for the $Q_{T}$-observed case, especially at small $Q_{T}$, where the bulk of dileptons is produced. For $Q_{T}$ smaller than the invariant mass $Q$ of the dilepton, soft gluon emissions contributing as $\alpha_{s}^{n} \log ^{m}\left(Q^{2} / Q_{T}^{2}\right) / Q_{T}^{2}(m \leq 2 n-1)$ bring dominant corrections in each order in $\alpha_{s}$. We perform all-order resummation of them at the next-to-leading logarithmic (NLL) accuracy, i.e., of the LL $(m=2 n-1)$ and NLL $(m=2 n-2,2 n-3)$ terms. The parton distributions at the low scale $Q_{T}$ can participate in $\mathcal{A}_{T T}\left(Q_{T}\right)$, while $A_{T T}$ in [2] is determined solely by the distributions at the scale $Q ; \mathcal{A}_{T T}\left(Q_{T}\right)$ may be larger than $A_{T T}$.

The spin-dependent part of the $Q_{T}$-differential cross section can be expressed as $[3,4]$

$$
\frac{\Delta_{T} d \sigma}{d Q^{2} d Q_{T}^{2} d y d \phi}=\cos (2 \phi) \frac{\alpha^{2}}{3 N_{c} S Q^{2}}\left[\Delta_{T} \tilde{X}^{\mathrm{NLL}}\left(Q_{T}^{2}, Q^{2}, y\right)+\Delta_{T} \tilde{Y}\left(Q_{T}^{2}, Q^{2}, y\right)\right]
$$

where $\sqrt{S}$ and $y$ denote the energy of the the incoming protons and rapidity of dilepton in the proton-proton CM system, and $\phi$ is the azimuthal angle of one of the outgoing leptons with respect to the proton's spin axis. The first term $\Delta_{T} \tilde{X}^{\mathrm{NLL}}$ denotes the NLL resummed cross section which is given by the integral over the impact parameter $b$, according to the general formalism of Collins-Soper-Sterman [5] combined with varous kinds of elaboration [3, 4, 6]:

$$
\begin{aligned}
& \Delta_{T} \tilde{X}^{\mathrm{NLL}}\left(Q_{T}^{2}, Q^{2}, y\right)=\int_{\mathcal{C}} d b \frac{b}{2} J_{0}\left(b Q_{T}\right) e^{S(b, Q)-g_{N P} b^{2}}\left[\delta H\left(x_{1}^{0}, x_{2}^{0} ; \frac{b_{0}^{2}}{b^{2}}\right)\right. \\
& \left.\quad+\frac{\alpha_{s}\left(Q^{2}\right)}{2 \pi}\left\{\int_{x_{1}^{0}}^{1} \frac{d z}{z} \Delta_{T} C_{q q}^{(1)}(z) \delta H\left(\frac{x_{1}^{0}}{z}, x_{2}^{0} ; \frac{b_{0}^{2}}{b^{2}}\right)+\int_{x_{2}^{0}}^{1} \frac{d z}{z} \Delta_{T} C_{q q}^{(1)}(z) \delta H\left(x_{1}^{0}, \frac{x_{2}^{0}}{z} ; \frac{b_{0}^{2}}{b^{2}}\right)\right\}\right]
\end{aligned}
$$

${ }^{*}$ Deceased. 
where $x_{1,2}^{0}=\sqrt{Q^{2} / S} e^{ \pm y}$ is the Drell-Yan scaling variables, $J_{0}\left(b Q_{T}\right)$ is a Bessel function, $b_{0}=2 e^{-\gamma_{E}}$ with the Euler's constant $\gamma_{E}$, and

$$
\delta H\left(x_{1}, x_{2} ; \mu^{2}\right)=\sum_{q} e_{q}^{2}\left[\delta q\left(x_{1}, \mu^{2}\right) \delta \bar{q}\left(x_{2}, \mu^{2}\right)+\delta \bar{q}\left(x_{1}, \mu^{2}\right) \delta q\left(x_{2}, \mu^{2}\right)\right] .
$$

Using $\lambda=\beta_{0} \alpha_{s}\left(Q^{2}\right) \log \left(Q^{2} b^{2} / b_{0}^{2}+1\right) \equiv \beta_{0} \alpha_{s}\left(Q^{2}\right) \tilde{L}$ with $\beta_{0}=\left(11 N_{c}-2 N_{f}\right) /(12 \pi)$, the large logarithmic corrections are resummed into the Sudakov factor $e^{S(b, Q)}=e^{h^{(0)}(\lambda) / \alpha_{s}\left(Q^{2}\right)+h^{(1)}(\lambda)}$, where $h^{(0)}(\lambda)=\left(A_{q}^{(1)} / 2 \pi \beta_{0}^{2}\right)[\lambda+\log (1-\lambda)]$ with $A_{q}^{(1)}=2 C_{F}=\left(N_{c}^{2}-1\right) / N_{c}$ collects the LL contributions, and $h^{(1)}(\lambda)$ corresponds to the NLL contributions; the explicit form of $h^{(1)}(\lambda)$, as well as another perturbatively calculable function $\Delta_{T} C_{q q}^{(1)}(z)$, is found in [3, 4]. $\tilde{L}$ plays a role of the large logarithmic expansion parameter in the $b$ space, as $b \sim 1 / Q_{T}$. We have introduced the Gaussian smearing factor $e^{-g_{N} b^{2}}$ in (2), with a nonperturbative parameter $g_{N P}[5]$, to take care of the long-distance behavior in the extremely large $|b|$ region. For the detail of elaboration of (2) beyond CSS, including the choice of the $b$-integration contour $\mathcal{C}$, see $[3,4]$. The second term in (1), $\Delta_{T} \tilde{Y}$, is of $\mathcal{O}\left(\alpha_{s}\right)$, and does not contain the singular terms to be resummed, such as $\sim \log \left(Q^{2} / Q_{T}^{2}\right) / Q_{T}^{2}$ and $1 / Q_{T}^{2} ; \Delta_{T} \tilde{Y}$ is determined [3] such that the expansion of $(1)$ to $\mathcal{O}\left(\alpha_{s}\right)$ reproduces the LO cross section for finite $Q_{T}$, which is of $\mathcal{O}\left(\alpha_{s}\right)$. Accordingly, we refer to (1) as the "NLL+LO" cross section. The NLL+LO cross section for unpolarized DY process is obtained similarly as (1), with $\tilde{X}^{\mathrm{NLL}}$ and $\tilde{Y}$ as the counterparts of $\Delta_{T} \tilde{X}^{\mathrm{NLL}}$ and $\Delta_{T} \tilde{Y}$, so that the NLL+LO asymmetry reads [4]

$$
\mathcal{A}_{T T}\left(Q_{T}\right)=\frac{\cos (2 \phi)}{2} \frac{\Delta_{T} \tilde{X}^{\mathrm{NLL}}\left(Q_{T}^{2}, Q^{2}, y\right)+\Delta_{T} \tilde{Y}\left(Q_{T}^{2}, Q^{2}, y\right)}{\tilde{X}^{\mathrm{NLL}}\left(Q_{T}^{2}, Q^{2}, y\right)+\tilde{Y}\left(Q_{T}^{2}, Q^{2}, y\right)} .
$$

In the following Figs. 1 and 2, we show the asymmetries $\mathcal{A}_{T T}\left(Q_{T}\right)$ for $\phi=0$, using a model of the NLO transversity distributions constructed as in [2], and $g_{N P}=0.5$ $\mathrm{GeV}^{2}$ for the nonperturbative parameter of (2). Figure 1 shows [4] the asymmetries at RHIC kinematics, $\sqrt{S}=200 \mathrm{GeV}, Q=5$ $\mathrm{GeV}$, and $y=2$. The solid line shows the NLL+LO result (4), the dot-dashed line shows the NLL result $\mathcal{A}_{T T}^{\mathrm{NLL}}\left(Q_{T}\right)$, obtained by omitting $\Delta_{T} \tilde{Y}$ and $Y$ in (4), and the two-dot-dashed line shows the LL result $\mathcal{A}_{T T}^{\mathrm{LL}}\left(Q_{T}\right)$, which is obtained by retaining only the LL terms in $\mathcal{A}_{T T}^{\mathrm{NLL}}\left(Q_{T}\right)$ :

$$
\mathcal{A}_{T T}^{\mathrm{LL}}\left(Q_{T}\right)=\frac{\cos (2 \phi)}{2} \frac{\delta H\left(x_{1}^{0}, x_{2}^{0} ; Q^{2}\right)}{H\left(x_{1}^{0}, x_{2}^{0} ; Q^{2}\right)}
$$

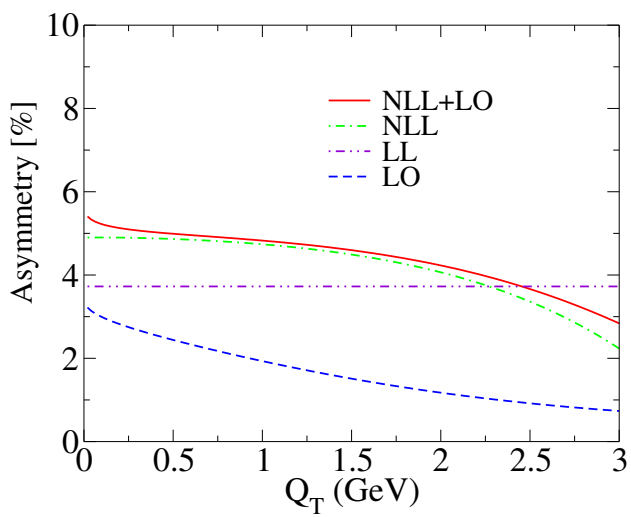

Figure 1: The asymmetries at RHIC, using (5) $\sqrt{S}=200 \mathrm{GeV}, Q=5 \mathrm{GeV}, y=2$ and $\phi=0$.

where $H$ is obtained from $\delta H$ of (3) by replacing $\delta q\left(x, \mu^{2}\right)$ with the density distributions $q\left(x, \mu^{2}\right)$, and (5) is independent of $Q_{T}$ [4]. The dashed line shows the LO asymmetry as the ratio of the LO cross sections. The NLL $+\mathrm{LO}$ result is flat and close to $\mathcal{A}_{T T}^{\mathrm{NLL}}\left(Q_{T}\right)$ in the small $Q_{T}$ region around $Q_{T} \simeq 1 \mathrm{GeV}$ : in this region, the NLL+LO cross section (1) and the corresponding unpolarized one are dominated by the resummed contributions $\Delta_{T} \tilde{X}^{\mathrm{NLL}}$ and 

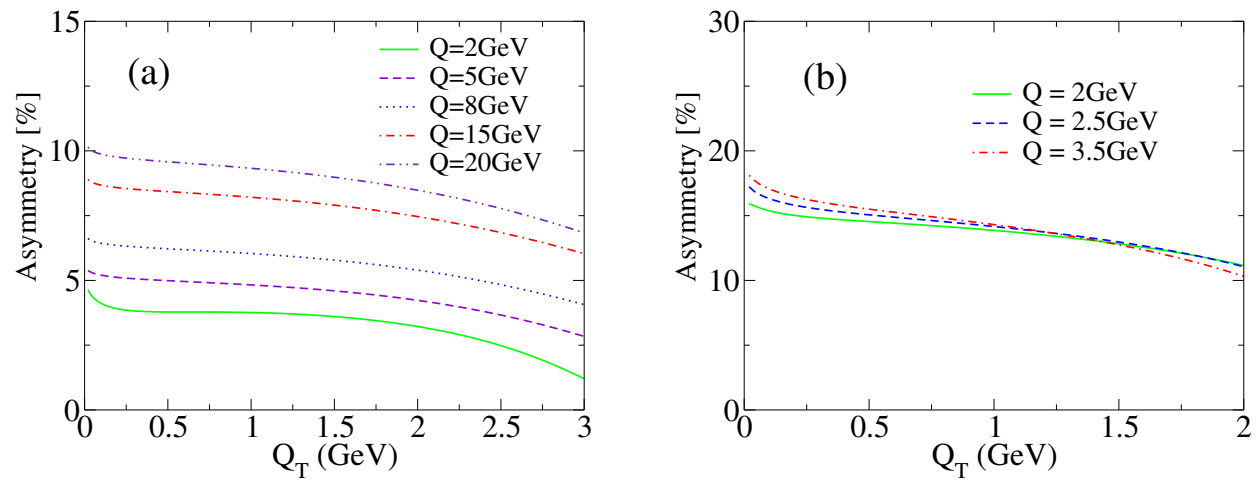

Figure 2: The NLL+LO asymmetries (4) for $\phi=0$ at (a) RHIC kinematics with $\sqrt{S}=200$ $\mathrm{GeV}$ and $y=2$, and (b) J-PARC kinematics with $\sqrt{S}=10 \mathrm{GeV}$ and $y=0$.

$\tilde{X}^{\mathrm{NLL}}$, and form a well-developed peak [3, 4]; moreover, the Sudakov factor $e^{S(b, Q)}$ of $(2)$ due to soft gluon resummation is universal up to the NLL level, so the dominant contributions cancel between $\Delta_{T} \tilde{X}^{\mathrm{NLL}}$ and $\tilde{X}^{\mathrm{NLL}}$ in $\mathcal{A}_{T T}^{\mathrm{NLL}}\left(Q_{T}\right)$. However, remarkably, some effects at the NLL level survive the cancellation, and raise $\mathcal{A}_{T T}^{\mathrm{NLL}}\left(Q_{T}\right)$ at small $Q_{T}$ significantly compared with $\mathcal{A}_{T T}^{\mathrm{LL}}\left(Q_{T}\right)$ that coincides with the conventional asymmetry $A_{T T}$ [2] using $Q_{T}$-integrated cross sections, up to the NLO $\left(\mathcal{O}\left(\alpha_{s}\right)\right)$ corrections. On the other hand, the LO result is much smaller than the other asymmetries and decreases as $Q_{T}$ increases, indicating that the soft gluon resummation is crucial for the prediction of the asymmetries.

The NLL+LO asymmetries $\mathcal{A}_{T T}\left(Q_{T}\right)$ of (4) at RHIC kinematics, $\sqrt{S}=200 \mathrm{GeV}, y=2$, and various values of $Q$, are presented in Fig. 2 (a), which shows that $\mathcal{A}_{T T}\left(Q_{T}\right)$ increases for increasing $Q$. This $Q$ dependence is a result of the small- $x$ behavior of the relevant parton distributions, in particular, the steep rise of the unpolarized sea-distributions for small $x_{1,2}^{0}=\sqrt{Q^{2} / S} e^{ \pm y}$, which enhances the denominator of (4) for small $Q$. Figure 2 (b) is same as Fig. 2 (a), but for possible polarized $p p$ experiment at J-PARC, $\sqrt{S}=10$ $\mathrm{GeV}, y=0$, and $Q=2,2.5,3.5 \mathrm{GeV}$, where the distributions at moderate $x$ are probed and $\mathcal{A}_{T T}\left(Q_{T}\right)$ at the flat region are around $15 \%$, irrespective of the value of $Q$. We find [4] that all cases of Figs. 2 (a) and (b) in fact obey the similar mechanism as shown in Fig. 1, resulting in the values of $\mathcal{A}_{T T}\left(Q_{T}\right)$ larger by $20-30 \%$ than the corresponding NLO $A_{T T}$.

The NLL+LO asymmetry (4) in the flat region as in Figs. 1, 2 can be generically approximated, to good accuracy, as $\mathcal{A}_{T T}\left(Q_{T}\right) \approx \mathcal{A}_{T T}^{\mathrm{NLL}}\left(Q_{T}=0\right)$, which is completely expressed by $\Delta_{T} \tilde{X}^{\mathrm{NLL}}$ and $\tilde{X}^{\mathrm{NLL}}$ at $Q_{T}=0$. The $b$-integration in those quantities can be evaluated analytically by the saddle-point method: for $(2)$ at $Q_{T}=0$, we get [4]

$$
\Delta_{T} \tilde{X}^{\mathrm{NLL}}\left(0, Q^{2}, y\right)=\left(\frac{b_{0}^{2}}{4 Q^{2} \beta_{0} \alpha_{s}\left(Q^{2}\right)} \sqrt{\frac{2 \pi}{\zeta^{\prime \prime}\left(\lambda_{S P}\right)}} e^{-\zeta\left(\lambda_{S P}\right)+h^{(1)}\left(\lambda_{S P}\right)}\right) \delta H\left(x_{1}^{0}, x_{2}^{0} ; b_{0}^{2} / b_{S P}^{2}\right),
$$

where $\zeta(\lambda)=-\lambda /\left(\beta_{0} \alpha_{s}\left(Q^{2}\right)\right)-h^{(0)}(\lambda) / \alpha_{s}\left(Q^{2}\right)+\left(g_{N P} b_{0}^{2} / Q^{2}\right) e^{\lambda /\left(\beta_{0} \alpha_{s}\left(Q^{2}\right)\right)}$, and $b_{S P}=$ $\left(b_{0} / Q\right) e^{\lambda_{S P} /\left(2 \beta_{0} \alpha_{s}\left(Q^{2}\right)\right)}$, with $\lambda_{S P}$ satisfying $\zeta^{\prime}\left(\lambda_{S P}\right)=0$, i.e.,

$$
1-\frac{A_{q}^{(1)}}{2 \pi \beta_{0}} \frac{\lambda_{S P}}{1-\lambda_{S P}}=\frac{g_{N P} b_{0}^{2}}{Q^{2}} e^{\frac{\lambda_{S P}}{\beta_{0} \alpha_{s}\left(Q^{2}\right)}} .
$$




\begin{tabular}{|c|r|r|r|r|r||r|r|r|}
\hline & \multicolumn{4}{|c||}{$\sqrt{S}=200 \mathrm{GeV}, y=2$} & \multicolumn{3}{c|}{$\sqrt{S}=10 \mathrm{GeV}, y=0$} \\
\hline$Q$ & $2 \mathrm{GeV}$ & $5 \mathrm{GeV}$ & $8 \mathrm{GeV}$ & $15 \mathrm{GeV}$ & $20 \mathrm{GeV}$ & $2 \mathrm{GeV}$ & $2.5 \mathrm{GeV}$ & $3.5 \mathrm{GeV}$ \\
\hline SP-I & $4.3 \%$ & $5.4 \%$ & $6.6 \%$ & $8.7 \%$ & $9.8 \%$ & $14.1 \%$ & $14.5 \%$ & $14.8 \%$ \\
SP-II & $7.3 \%$ & $8.7 \%$ & $9.8 \%$ & $11.8 \%$ & $12.7 \%$ & $14.7 \%$ & $14.8 \%$ & $14.2 \%$ \\
\hline
\end{tabular}

Table 1: $\mathcal{A}_{T T}^{\mathrm{NLL}}\left(Q_{T}=0\right)$ for $\phi=0$ using the saddle-point formula (8).

Here (6) gives the saddle-point formula in the NLL accuracy, and corresponds to extension of that in the LL level in the literature [5]: the solution of (7) formally determines the saddle point at the LL level combined with the contribution due to the Gaussian factor $e^{-g_{N P} b^{2}}$ in (2), but we find [4] that the "shift" of the saddle point at the NLL level from $\lambda_{S P}$ yields only the NNLL corrections to (6); note that the NNLL contributions are of $\mathcal{O}\left(\alpha_{s}\right)$, according to the counting of the relevant logarithms in the region $Q_{T} \approx 0$ (see also [5]). The saddle-point formula for $\tilde{X}^{\mathrm{NLL}}\left(0, Q^{2}, y\right)$ can be obtained similarly, and the result is given by the above result (6) with the replacement $\delta H\left(x_{1}^{0}, x_{2}^{0} ; b_{0}^{2} / b_{S P}^{2}\right) \rightarrow H\left(x_{1}^{0}, x_{2}^{0} ; b_{0}^{2} / b_{S P}^{2}\right)$. The common factor, in the parentheses of (6), involves "very large perturbative effects" due to the universal Sudakov factor, but this factor cancels out for the asymmetry. We get [4]

$$
\mathcal{A}_{T T}^{\mathrm{NLL}}\left(Q_{T}=0\right)=\frac{\cos (2 \phi)}{2} \frac{\delta H\left(x_{1}^{0}, x_{2}^{0} ; b_{0}^{2} / b_{S P}^{2}\right)}{H\left(x_{1}^{0}, x_{2}^{0} ; b_{0}^{2} / b_{S P}^{2}\right)},
$$

which is exact up to the NNLL $\left(\mathcal{O}\left(\alpha_{s}\right)\right)$ corrections for $Q_{T} \approx 0$. This clarifies the mechanism discussed in Fig. 1: the contributions surviving the cancellation in (8) are entirely absorbed into the unconventional scale $b_{0} / b_{S P}$ for the relevant distribution functions. Compared with (5), participation of the new scale $b_{0} / b_{S P}$ is the effect at the NLL level, and, remarkably, $b_{0} / b_{S P}$ using (7) depends weakly on $Q$, as $b_{0} / b_{S P} \simeq 1 \mathrm{GeV}$ for all cases in Figs. 1 and 2 [4]. This explains why $\mathcal{A}_{T T}\left(Q_{T}\right)$ at small $Q_{T}$ is always larger than (5), or the NLO $A_{T T}$ in [2]. Also $\mathcal{A}_{T T}^{\mathrm{NLL}}\left(Q_{T}\right)$ does not approach to $\mathcal{A}_{T T}^{\mathrm{LL}}\left(Q_{T}\right)$ even in the $Q \rightarrow \infty$ limit.

In Table 1, both "SP-I" and "SP-II" show $\mathcal{A}_{T T}^{\mathrm{NLL}}\left(Q_{T}=0\right)$ using $(8)$ with $(7)$, but these two cases differ by the contributions at the NNLL level, reflecting mismatch to classify the terms between NLL and NLO (for the detail of SP-I, II, see [4]). SP-I reproduces $\mathcal{A}_{T T}\left(Q_{T}=0\right.$ ) in the flat region in Fig. 2 to the $10 \%$ accuracy, i.e., to the canonical size of $\mathcal{O}\left(\alpha_{s}\right)$ corrections associated with the NLL accuracy. However, SP-II overestimates for RHIC, demonstrating that certain NNLL corrections would grow at the small- $x$ region, the edge region of the phase space, beyond the canonical size. To this accuracy, our simple formula (8) is applicable in order to extract the NLO transversity distributions directly from the data.

This work was supported by the Grant-in-Aid for Scientific Research No. B-19340063.

\section{References}

[1] Slides:

http://indico. cern. ch/contributionDisplay . py? contribId=159\&sessionId=4\&conf Id=9499

[2] O. Martin et al., Phys. Rev. D57 3084 (1998); ibid. D60 117502 (1999).

[3] H. Kawamura, J. Kodaira, H. Shimizu and K. Tanaka, Prog. Theor. Phys. 115, 667 (2006).

[4] H. Kawamura, J. Kodaira and K. Tanaka, arXiv:hep-ph/0703079, to appear in Nucl. Phys. B (2007).

[5] J. C. Collins, D. Soper and G. Sterman, Nucl. Phys. B250 199 (1985).

[6] G. Bozzi et al., Phys. Lett. B564 65 (2003); Nucl. Phys. B737 573 (2006); arXiv:0705.3887 [hep-ph]. 